\title{
Protocol for a systematic review and meta-analysis of the diagnostic accuracy of artificial intelligence for grading of ophthalmology imaging modalities
}

Jessica Cao ( $\sim$ jessica.cao@mail.utoronto.ca )

University of Toronto https://orcid.org/0000-0001-6073-8814

Glen Katsnelson

University of Toronto

Brittany Chang-Kit

University of Toronto

Parsa Merhraban Far

Queen's University

Elizabeth Uleryk

E/M Consulting

Adeteju Ogunbameru

University of Toronto

Rafael Neves Miranda

University of Toronto

Tina Felfeli

University of Toronto https://orcid.org/0000-0002-0927-3086

\section{Research Article}

Keywords: ophthalmology, artificial intelligence, diagnostic accuracy, image grading, meta-analysis

Posted Date: January 18th, 2022

DOI: https://doi.org/10.21203/rs.3.rs-1240371/v1

License: (c) (i) This work is licensed under a Creative Commons Attribution 4.0 International License.

Read Full License 


\section{Abstract}

\section{Background}

With the rise of artificial intelligence (Al) in ophthalmology, the need to define its diagnostic accuracy is increasingly important. The review aims to elucidate the diagnostic accuracy of Al algorithms in screening for all ophthalmic conditions in patient care settings that involve digital imaging modalities, using the reference standard of human graders.

\section{Methods}

This is a systematic review and meta-analysis. A literature search will be conducted on Ovid Medline, Ovid EMBASE, and Wiley Cochrane CENTRAL from January 1, 2000 to December 20, 2021. Studies will be selected via screening titles and abstracts, followed by full-text screening. Articles that compare the results of Al-graded ophthalmic images with results from human graders as a reference standard will be included. Systematic review software DistillerSR will be used to automate part of the screening process as an adjunct to human reviewers. After full text screening, data will be extracted from each study via the categories of study characteristics, patient information, Al methods, intervention, and outcomes. Risk of bias will be scored using Quality Assessment of Diagnostic Accuracy Studies (QUADAS-2) by two trained independent reviewers. Disagreements at any step will be addressed by a third adjudicator. Study results will include summary receiver operating characteristic (SROC) curve plots as well as pooled sensitivity and specificity of artificial intelligence for detection of any ophthalmic conditions based on imaging modalities compared to the reference standard. Statistics will be calculated in R statistical software.

\section{Discussion}

This study will provide novel insights on diagnostic accuracy of Al in new domains of ophthalmology that have not been previously studied. The protocol also outlines the use of an Al-based software to assist article screening, which may serve as a reference for improving efficiency and accuracy of future large systematic reviews.

Systematic Review Registration: This study is registered on PROSPERO (CRD42021274441).

\section{Background}

Imaging is an important diagnostic and prognostic tool in ophthalmic patient care (1). With everincreasing use of diagnostic imaging in technology, there is a growing need for accurate and efficient grading of ophthalmic images for informing patient care. Much research has been done in recent years into artificial intelligence (Al) systems that can analyze ophthalmic images and provide an accurate screening result $(2,3)$. With the increasing number of patients screened through teleophthalmology, there is a growing demand for experienced human graders such as subspecialty ophthalmologists (4-6). Deep learning has already shown promise in ophthalmic image recognition capabilities (7). Previous 
systematic reviews have shown that Al has a sensitivity of $80-100 \%$ and specificity of $84-100 \%$ in diagnosing diabetic retinopathy, a condition involving the posterior segment of the eye, from fundus photographs $(8,9)$. The use of $\mathrm{Al}$ in anterior segment diseases has been also explored more recently in a multicenter study, which suggested a sensitivity and specificity of $89.7 \%$ and $86.4 \%$, respectively, in the diagnosis of pediatric cataract (10). Advancements in automated image analysis are valuable to eye disease screening programs, particularly those in day-to-day disease risk prediction and virtual care such as teleophthalmology, which aim to reduce barriers to care, particularly in underserved populations (11). Machine learning models such as convolutional neural networks are used in medical image analysis to automate recognition and diagnosis (12).

Published systematic reviews on the topic of diagnostic accuracy of Al for grading ophthalmic images have had a narrow scope limited to a few ophthalmic conditions such as diabetic retinopathy, age-related macular degeneration, glaucoma, and retinopathy of prematurity $(7,13)$. Previous studies have recognized the potential of Al for use in other applications in ophthalmology but this information has yet to be synthesized and reviewed critically (9). More information is needed on the specific Al tools available, as well as their reliability in providing accurate diagnoses in all clinical contexts of ophthalmology including assistance in clinical decision-making (14).

Given the rise of Al in medicine and ophthalmology, defining its accuracy, and reliability, will guide future research in this area and enhance its real-life adaption. This review aims to elucidate the diagnostic accuracy of artificial intelligence in screening for all ophthalmic conditions in patient care settings that involve digital imaging modalities, using the reference standard of human graders.

\section{Methods}

\section{Study design}

This is a systematic review and meta-analysis. This protocol is registered in PROSPERO (CRD42021274441).

\section{Study Objectives}

This project aims to determine the diagnostic accuracy of Al in ophthalmology clinical settings, with results stratified by and presented for each ophthalmic condition. Where sufficient information is available, patients will also be grouped by age, either pediatric (under 18 years of age) or adult (18 years or older). Some ophthalmic conditions, such as retinopathy of prematurity, occur exclusively in the pediatric population, whereas others such as age-related macular degeneration occur most commonly in senior adults. Studies with a mix of patient ages will be characterized based on the proportion of adult and pediatric patients. Both these examples provide potential for Al-assisted screening through automated grading of various diagnostic imaging modalities. 
The present study will further subgroup ophthalmic conditions by their anatomic location. Anterior segment conditions include cataract, keratoconus, and dry eye disease. Common forms of imaging include anterior segment optical coherence tomography (AS-OCT), keratometry, and slit lamp photography. Posterior segment conditions such as diabetic retinopathy, age-related macular degeneration, and open angle glaucoma can be visualized via imaging modalities such as OCT of the macular and optic nerve, fundus photography, and visual field testing.

Additional subgroups for studies will be based on the setting of clinic or remote via teleophthalmology. This will allow authors to discern whether patient setting is related to the diagnostic accuracy of Al.

For all analyses, human graders will serve as the reference standard and will assess the diagnostic accuracy of the Al screening results relative to images graded by humans. Human graders were set as our reference standard as human grading is the predominant and best method thus far in providing a diagnosis. As diagnoses can differ between eyes for each individual, this study will use the eye as the unit of analysis.

\section{Search strategy}

We will undertake a literature search of relevant articles using a comprehensive search strategy developed in consultation with experienced librarians. The search will be conducted on Ovid Medline, Ovid EMBASE, and Wiley Cochrane CENTRAL for articles from January 1, 2000 to December 20, 2021. The timeline of 2000 as the initial search start date was chosen to reflect the recency of Al development and application, including one of the first studies using Al in ophthalmology, which was published in 2004 (15). The search will include a group of terms related to artificial intelligence and ophthalmology. Subject headings as well as key terms will be included. The search was first developed on Ovid Medline, then translated to Ovid EMBASE and Wiley Cochrane CENTRAL. The search will not be restricted based on language or patient population. Supplementary Data 1 includes the complete search strategy for all three databases.

\section{Study Selection}

\section{Inclusion and Exclusion criteria}

Peer-reviewed scientific articles found in the chosen databases that compare the results of Al-graded ophthalmic images with results from human graders will be included. The scope of imaging for ophthalmic conditions will include, but are not limited to keratoconus, cataract, angle-closure glaucoma, dry eye disease, posterior capsule opacification, diabetic retinopathy, age-related macular degeneration, retinopathy of prematurity, open-angle glaucoma, epiretinal membrane, and macular hole. Patients of any age or comorbidity status will be included.

Review papers, case reports, conference abstracts, guidelines, editorials, commentaries, and opinion pieces will be excluded. Papers not in English will be excluded.

\section{Softwares Used}


Due to the large number of anticipated studies from the search, the systematic review software DistillerSR (Evidence Partners) was chosen to assist with de-duplication of citations and screening of articles (16). DistillerSR uses machine learning to automate part of the screening process as an adjunct to human graders (17). After providing the software with a training set where reviewers manually provide the screening result, DistillerSR software will recognize patterns and keywords used for screening that can be applied to the remainder of articles. A relevance threshold level can be set to control the strictness of screening, and manual checks are available at various steps to ensure a desired screening result. Using this software will allow a much broader scope to be accomplished than previous systematic reviews on the topic.

All statistical analysis for the meta-analysis will be completed with $\mathrm{R}$.

\section{Screening of Studies}

Retrieved studies from the searched databases will be imported into the systematic review software DistillerSR and deduplicated. Studies will be selected via a two-stage screening process, first by screening titles and abstracts, followed by full-text screening. The screening process will be supplemented with DistillerSR using a stepwise approach. After undergoing training on inclusion and exclusion criteria, two independent reviewers will screen papers until a minimum of 10 relevant articles selected for inclusion and a total of 500 articles screened is reached. This will serve as the training set for the automated DistillerSR screening software. For the next set of 500 articles, one reviewer will screen titles and abstracts and DistillerSR will be used as the second reviewer. These thresholds were chosen as a conservative approach to screening based on manufacturer recommendations for optimal performance of the software. A relevance threshold will be set at 0.1 (most conservative threshold chosen to ensure high sensitivity for inclusion of studies and prevent exclusion of any relevant articles). If an acceptable level of agreement (>90\%) between the reviewer and DistillerSR is achieved, the remaining set of articles will be graded by DistillerSR alone (18). In this case, a quality check of a random selection of $10 \%$ of articles screened by DistillerSR alone will be done by a reviewer to ensure no relevant studies are excluded. 4) In case an acceptable level of agreement is not achieved ( $<90 \%)$, the algorithm will be re-run with the inclusion of newly screened articles to increase the training set size. Below the relevance threshold of 0.1 , we will use DistillerSR only for screening. If again the level of agreement is $<90 \%$, then one reviewer will screen the papers with DistillerSR serving as the second screener. In all steps, any disagreements will be reviewed by a third senior adjudicator.

\section{Data Extraction}

After full text screening, data will be extracted from each study via the categories of study characteristics, patient information, Al methods, and outcomes (e.g. sensitivity and specificity). The full list of data categories to be extracted is presented in Table 1. 


\begin{tabular}{|c|c|}
\hline Data Category & Collected data \\
\hline \multirow{12}{*}{$\begin{array}{l}\text { Study } \\
\text { characteristics }\end{array}$} & - Primary author \\
\hline & - Publication year \\
\hline & - Recruitment period/study duration \\
\hline & - Country \\
\hline & - Study purpose \\
\hline & - Study type (eg. RCT, prospective cohort study) \\
\hline & - Sample size \\
\hline & - Clinical setting (academic/community) \\
\hline & - Reference standard description (eg. human graders - retina specialists) \\
\hline & - Ophthalmic condition screened for \\
\hline & - Funding sources \\
\hline & - Follow-up period \\
\hline \multirow[t]{2}{*}{$\begin{array}{l}\text { Patient } \\
\text { information }\end{array}$} & $\begin{array}{l}\text { - Patient sociodemographic data (including age (mean/median and categorization } \\
\text { of pediatric and adult), sex, comorbidities, eye conditions, race/ethnicity, income } \\
\text { status, education) }\end{array}$ \\
\hline & - Inclusion and exclusion criteria \\
\hline \multirow[t]{6}{*}{ Al Methods } & $\begin{array}{l}\text { - Imaging modalities used for screening (e.g. fundus photographs, ocular coherence } \\
\text { tomography) }\end{array}$ \\
\hline & - Automated algorithms or tools used (boosted tree, random forest, etc) \\
\hline & - Role of Al in screening \\
\hline & - Number of human graders \\
\hline & - Number of ungradable images \\
\hline & - Identified pathologies (types and proportions) \\
\hline \multirow{5}{*}{$\begin{array}{l}\text { Intervention } \\
\text { Outcomes }\end{array}$} & - Sensitivity/specificity \\
\hline & - Positive predictive value \\
\hline & - Negative predictive value \\
\hline & - \% correct as analyzed by artificial intelligence \\
\hline & - Diagnostic accuracy (if stated) \\
\hline
\end{tabular}


Quality Assessment of Diagnostic Accuracy Studies (QUADAS-2) will be used by two independent reviewers to assess the quality of included studies based on the 4 domains of index test, reference test, patient selection and flow/timing (19). Multiple signalling questions for each domain guide the bias review. Risk of bias is graded as high, low, or unclear. A grading of unclear is given only if there is insufficient information to make a decision. If at least one signalling question is answered as "no", there is potential for bias and reviewers will independently judge the risk for bias. Unclear grading results when there is insufficient data for a judgement to be made.

In cases where studies exclude patients from the comparative analysis, we established a low risk-of-bias cut-off at $10 \%$ of ophthalmic images that were deemed ungradable by the human graders. This cut-off was informed by a selection of review papers, which labelled a $5-10 \%$ ungradable rate as low $(20,21)$.

Any disagreements in grading will be reviewed by a third adjudicator. A summary and graphic representation of the QUADAS-2 gradings for all studies will be presented in the final review. A sensitivity analysis will be conducted by removing studies with a high risk of bias.

\section{Missing Data}

Where there is missing data, we will make attempts to contact the corresponding author of studies through the email listed on the publication. A total of three attempts will be made. If no response is received, the authors will make the best attempt to perform the analysis based on available data and code any data not available as missing. The missing data will be noted as a limitation in the discussion section of the manuscript.

\section{Data synthesis}

For each study, screening outcomes via artificial intelligence will be entered in a two-by-two table (true positive, false positive, true negative, false negative). The data of the two-by-two tables will be used to calculate sensitivity and specificity for each study (Table 2). We will present individual study results graphically by plotting the estimates of sensitivity and specificity in both forest plots and on the summary receiver operating characteristic (SROC) curve plots. The predictive accuracy will be quantified using the AUROC.

Table 2

Sample Two-by-Two Contingency Table Used for Analysis

\begin{tabular}{|llll|}
\hline & & \multicolumn{2}{l|}{ Reference (result by human graders) } \\
\cline { 2 - 4 } Test (Al result) & Positive & Negative \\
\cline { 2 - 4 } & Positive & True Positive & False Positive \\
\cline { 2 - 4 } & Negative & False Negative & True Negative \\
\hline
\end{tabular}

We will also conduct a subgroup analysis on the diagnostic accuracy of artificial intelligence when used specifically in teleophthalmology programs. 
Our unit of analysis is the eye, given that each eye may have a separate diagnosis and therefore affect accuracy in different ways. Some studies may only report results per patient instead of per eye. As such, a sensitivity analysis will be conducted with the unit of analysis as each patient to ensure consistency of results.

Pooled sensitivity and specificity of artificial intelligence for detection of any ophthalmic conditions based on imaging modalities compared to the reference standard (i.e. human graders) will be reported. The findings will be stratified by ophthalmic condition (anterior vs posterior segment disease entities; when sufficient data is available), as well as demographics (pediatric vs adults $>=18$ years old). Pooled estimates of the sensitivity and specificity will be obtained with random effect models, using the DerSimonian-Laird method to incorporate variation among studies (22).

We will investigate heterogeneity firstly through visual examination of forest plots of sensitivities and specificities, as well as the SROC plot of the raw data. Last, we will use Cochran's $Q$ test to evaluate homogeneity. We will also use the statistic $I^{2}$ of Higgins to quantify the amount of heterogeneity. The scale of $\mathrm{I}^{2}$ has a range of 0 to $100 \%$ and values of $25 \%, 50 \%$, and $75 \%$ are considered low, moderate, and high heterogeneity, respectively. All statistical analyses will be completed by a qualified biostatistician.

\section{Discussion}

This systematic review will aim to identify the diagnostic accuracy of Al in image recognition for ophthalmic diseases. This will be the first review to our knowledge with a broad scope with no restriction on the type of ophthalmic condition, which will allow a thorough assessment of Al accuracy and reliability. The study findings can help clinicians to ascertain as to whether certain types of image analysis and screening can be allocated to Al systems, thus reducing healthcare resource utilization.

Al and automated image grading are most commonly used in some posterior segment pathologies such as diabetic retinopathy and age-related macular degeneration. Accordingly, we expect to find many studies on these conditions and have enough data to calculate a pooled sensitivity and specificity via meta-analysis. However, the use of Al has more recently been explored in other ophthalmic conditions. As such, data may be insufficient or show that current Al systems still need refinement before making reliable diagnoses. Similarly, the body of research on adult ophthalmic conditions is much wider compared to pediatric conditions. As such, we anticipate more Al systems as well as better diagnostic accuracy in adult ophthalmic pathologies due to the higher availability of training data.

We also anticipate differences to be noted in the diagnostic accuracy of Al based on the setting of use. Clinic settings have the additional benefit of the patient being present for a full history and clinical exam. Clinicians can rely on ancillary information to make a diagnosis, and thus the grading result of specific ophthalmic imaging may be less applicable. However, in the context of remote screening via teleophthalmology, human graders can only rely on limited clinical information in addition to imaging. Thus, Al may be able to play a more important role in teleophthalmology programs. 
There are some limitations to this study. Firstly, given that the quality and quantity of data in less common conditions is unknown, we may not be able to conduct an accurate meta-analysis and provide pooled sensitivity and specificity values for some diseases. Other potential limitations of the study include the reliability of human graders which serve as the reference standard. In our analysis the assumption is made that human graders have $100 \%$ sensitivity and specificity. There may be certain scenarios where human graders are incorrect or unable to provide a grading, and it will be difficult to determine this value from study to study. There is also a high variability in how results are reported by various studies. For example, some studies may report diagnosis of the ophthalmic condition at any severity, at a specific severity, or at referable disease, of which the definition may also vary (23). Due to the novelty of this topic, we anticipate only a small numbers of randomized control trials, which is typically believed to provide the highest level of evidence (24).

Overall, this systematic review and meta-analysis will provide novel insights on the diagnostic accuracy of Al in new domains of ophthalmology that have not been previously studied. Results from our review may help to either support the use of $\mathrm{Al}$ in specific applications in ophthalmology or point out areas of weakness in which Al lacks the reliability to be used in lieu of human graders. This protocol also documents use of an Al-based software to assist article screening, which may serve as a reference for future large systematic reviews to make screening more accessible.

\section{Declarations}

Ethics approval and consent to participate: Not applicable

Consent for publication: Not applicable

Availability of data and materials: Not applicable

Competing interests: The authors declare they have no competing interests.

Funding: No funding sources.

Authors' contributions: TF conceived the study. All authors contributed equally to the study design and drafting of the protocol. The search strategy was developed by JC, BC, GK, EU, and TF. All authors edited and approved the final manuscript.

Acknowledgements: The authors would like to acknowledge our librarian Elena Springall for her contribution in reviewing the search strategy.

\section{References}

1. Müller PL, Wolf S, Dolz-Marco R, Tafreshi A, Schmitz-Valckenberg S, Holz FG. Ophthalmic Diagnostic Imaging: Retina. In: High Resolution Imaging in Microscopy and Ophthalmology. 2019. 
2. Korot E, Wood E, Weiner A, Sim DA, Trese M. A renaissance of teleophthalmology through artificial intelligence. Vol. 33, Eye (Basingstoke). 2019.

3. Olvera-Barrios A, Heeren TFC, Balaskas K, Chambers R, Bolter L, Egan C, et al. Diagnostic accuracy of diabetic retinopathy grading by an artificial intelligence-enabled algorithm compared with a human standard for wide-field true-colour confocal scanning and standard digital retinal images. British Journal of Ophthalmology. 2021;105(2).

4. Keunen JEE, Verezen CA, Imhof SM, van Rens GHMB, Asselbergs MB, Limburg JJH. [Increase in the demand for eye-care services in the Netherlands 2010-2020]. Nederlands tijdschrift voor geneeskunde. 2011;155(41).

5. Yuan M, Chen W, Wang T, Song Y, Zhu Y, Chen C, et al. Exploring the growth patterns of medical demand for eye care: a longitudinal hospital-level study over 10 years in China. Annals of Translational Medicine. 2020;8(21).

6. Buchan JC, Norman P, Shickle D, Cassels-Brown A, MacEwen C. Failing to plan and planning to fail. Can we predict the future growth of demand on UK Eye Care Services? Vol. 33, Eye (Basingstoke). 2019.

7. Aggarwal R, Sounderajah V, Martin G, Ting DSW, Karthikesalingam A, King D, et al. Diagnostic accuracy of deep learning in medical imaging: a systematic review and meta-analysis. Vol. 4: npj Digital Medicine; 2021.

8. Islam MM, Yang HC, Poly TN, Jian WS, (Jack) Li YC. Deep learning algorithms for detection of diabetic retinopathy in retinal fundus photographs: A systematic review and meta-analysis. Computer Methods and Programs in Biomedicine. 2020;191.

9. Nielsen KB, Lautrup ML, Andersen JKH, Savarimuthu TR, Grauslund J. Deep Learning-Based Algorithms in Screening of Diabetic Retinopathy: A Systematic Review of Diagnostic Performance. Vol. 3, Ophthalmology Retina. 2019.

10. Lin H, Li R, Liu Z, Chen J, Yang Y, Chen H, et al. Diagnostic Efficacy and Therapeutic Decision-making Capacity of an Artificial Intelligence Platform for Childhood Cataracts in Eye Clinics: A Multicentre Randomized Controlled Trial. EClinicalMedicine. 2019;9.

11. Sharafeldin N, Kawaguchi A, Sundaram A, Campbell S, Rudnisky C, Weis E, et al. Review of economic evaluations of teleophthalmology as a screening strategy for chronic eye disease in adults. British Journal of Ophthalmology. 2018;102(11).

12. Yadav SS, Jadhav SM. Deep convolutional neural network based medical image classification for disease diagnosis. Journal of Big Data. 2019;6(1).

13. Wang S, Zhang Y, Lei S, Zhu H, Li J, Wang Q, et al. Performance of deep neural network-based artificial intelligence method in diabetic retinopathy screening: A systematic review and metaanalysis of diagnostic test accuracy. Vol. 183, European Journal of Endocrinology. 2020.

14. Kapoor R, Walters SP, Al-Aswad LA. The current state of artificial intelligence in ophthalmology. Vol. 64, Survey of Ophthalmology. 2019. 
15. Güler NF, Übeyli ED. Wavelet-based neural network analysis of ophthalmic artery Doppler signals. Computers in Biology and Medicine. 2004;34(7).

16. Evidence Partners. DistillerSR [Internet]. [cited 2021 Oct 12]. Available from: https://www.evidencepartners.com.

17. Read K, Husson H, Dobbins M. Can artificial intelligence learn to identify systematic reviews on the effectiveness of public health interventions? In: Advances in Evidence Synthesis: special issue. 2020.

18. Graham M, Milanowski A, Miller J. Measuring. and Promoting Inter-Rater Agreement of Teacher and Principal Performance Ratings [Internet]. 2012 [cited 2021 Oct 9]. Available from: https://files.eric.ed.gov/fulltext/ED532068.pdf.

19. University of Bristol. QUADAS-2 [Internet]. [cited 2021 Oct 16]. Available from: https://www.bristol.ac.uk/population-health-sciences/projects/quadas/quadas-2/.

20. Liu Y, Torres Diaz A, Benkert R. Scaling Up Teleophthalmology for Diabetic Eye Screening: Opportunities for Widespread Implementation in the USA. Vol. 19, Current Diabetes Reports. 2019.

21. Newman Casey PA. Telemedicine and Diabetic Retinopathy: Review of Published Screening Programs. Journal of Endocrinology and Diabetes. 2015;2(4).

22. Natarajan S, Jain A, Krishnan R, Rogye A, Sivaprasad S. Diagnostic Accuracy of Community-Based Diabetic Retinopathy Screening with an Offline Artificial Intelligence System on a Smartphone. JAMA Ophthalmology. 2019;137(10).

23. Hariton E, Locascio JJ. Randomised controlled trials - the gold standard for effectiveness research: Study design: randomised controlled trials. Vol. 125: BJOG: An International Journal of Obstetrics and Gynaecology; 2018.

\section{Supplementary Files}

This is a list of supplementary files associated with this preprint. Click to download.

- SupplementaryData1SearchStrategy.docx 